\title{
Gene Expression Changes Underlying Idiopathic Central Hypogonadism in Cryptorchidism with Defective Mini-Puberty
}

\author{
Faruk Hadziselimovic ${ }^{a}$ Katharina Gegenschatz-Schmid ${ }^{a} \quad$ Gilvydas Verkauskas $^{\mathrm{e}}$ \\ Maria J. Docampo-Garcia ${ }^{a}$ Philippe Demougin $^{b}$ Vytautas Bilius $^{\mathrm{e}}$ \\ Dalius Malcius ${ }^{g}$ Darius Dasevicius ${ }^{f}$ Michael B. Stadtler ${ }^{c, d}$

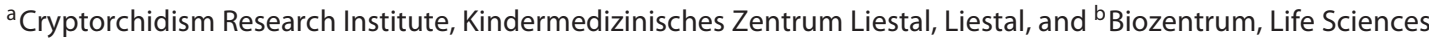 \\ Training Facility, University of Basel, ' $F$ riedrich Miescher Institute for Biomedical Research, and ${ }^{\mathrm{d}}$ Swiss Institute \\ of Bioinformatics, Basel, Switzerland; ${ }^{e}$ Children's Surgery Centre, Vilnius University, and ${ }^{f}$ Institute for Pathology, \\ National Centre of Pathology, Vilnius, and ${ }^{9}$ Lithuanian University of Health Sciences, Kaunas, Lithuania
}

\section{Key Words}

Ad spermatogonia - Cryptorchidism - Idiopathic central hypogonadism · Infertility · Mini-puberty · PROK2 .

RNA sequencing

\begin{abstract}
The whole genome RNA profiling of testicular biopsies by DNA strand-specific RNA sequencing was examined to determine a potential causative role of isolated congenital cryptorchidism in azoospermia and/or infertility in the context of our previously published GeneChip data. Cryptorchid patients, aged 7 months to 5 years and otherwise healthy, were enrolled in this prospective study. During surgery, testicular tissue biopsies were obtained for histological examination and RNA sequencing. Fifteen patients were selected based on the histological results and were divided into 2 groups. Seven were classified as belonging to the high infertility risk (HIR) and 8 to the low infertility risk (LIR) group. Cryptorchid boys in the HIR group lacked transformation of gonocytes into Ad spermatogonia due to impaired mini-puberty. This group of patients will be infertile despite success-
\end{abstract}

\section{KARGER}

E-Mail karger@karger.com www.karger.com/sxd

\section{(C) 2016 The Author(s) \\ Published by S. Karger AG, Basel $1661-5425 / 16 / 0103-0136 \$ 39.50 / 0$} This article is licensed under the Creative Commons Attribution-
NonCommercial-NoDerivatives 4.0 International License (CC BYNC-ND) (http://www.karger.com/Services/OpenAccessLicense) Usage and distribution for commercial purposes as well as any distribution of modified material requires written permission. ful surgery. The new important finding was a decreased PROK2, CHD7, FGFR1, and SPRY4 gene expression in the HIR group. Furthermore, identification of multiple differences in gene expression between HIR and LIR groups underscores the importance of an intact hypothalamic-pituitary-gonadal axis for fertility development. Our RNA profiling data strongly support the theory that in the HIR group of cryptorchid boys insufficient PROK2/CHD7/FGFR1/SPRY4 gene expression induces deficient $\mathrm{LH}$ secretion, resulting in impaired mini-puberty and infertility. We therefore recommend hormonal treatment for this cohort of cryptorchid boys with defective mini-puberty following a seemingly successful orchidopexy.

(c) 2016 The Author(s)

Published by S. Karger AG, Basel

Idiopathic central hypogonadism (ICH), with or without anosmia, is a disorder characterized by absent or incomplete sexual maturation by the age of 18 in conjunction with low levels of circulating gonadotropins and testosterone and no other abnormalities of the hypothalamic-pituitary axis [Bonomi et al., 2012]. Genetic inves- 
tigations of a human disease model of isolated gonadotropin-releasing hormone (GnRH) deficiency have revealed several key genes crucial for GnRH neuronal ontogeny and GnRH secretion [Martin et al., 2010]. Among these, PROK2 has emerged as a critical regulator of human reproduction [Martin et al., 2010]. In mammals, a unique network within the hypothalamus initiates and maintains reproductive function by virtue of $\mathrm{GnRH}$, which acts as a key messenger on the anterior pituitary gland [Antunes et al., 1978; Merchenthaler et al., 1984; Wray and Hoffman, 1986]. GnRH bound to receptors on pituitary gonadotrope cells stimulates the synthesis and release of luteinizing hormone (LH) and follicle-stimulating hormone (FSH). Furthermore, each of the transcription factors that recognize $\mathrm{LH}$ promoter binding sites (SF1, EGR1, and PITX1) can function alone or in synergy with others to activate the LH promoter through direct physical interaction [Tremblay and Drouin, 1999].

Cryptorchidism is the most frequent congenital disorder in newborn boys [Miller et al., 2009; Chung and Brock, 2011]. Noticeably, cryptorchidism represents the most common cause of nonobstructive azoospermia in man [Fedder et al., 2004]. During a period of between 30 and 90 days after birth, activation of the hypothalamic-pituitary-gonadal (HPG) axis leads to a transient increase of gonadotropins and testosterone [Forest et al., 1974; Winter et al., 1976; Corbier et al., 1992]. Over the course of this time period, which in 2004 we termed mini-puberty, the transformation of gonocytes into A dark (Ad) spermatogonia occurs in a testosterone-dependent manner [Hadziselimovic et al., $2004,2005]$. Fifteen years ago, impaired secretion of LH and testosterone during mini-puberty was claimed to be the main reason for cryptorchidism-induced infertility [Hadziselimovic et al., 2004, 2005]. As a consequence of defective mini-puberty, the transformation of Ad spermatogonia was abolished (high infertility risk group, HIR), resulting in infertility after puberty despite early and successful surgery [Hadziselimovic and Hoecht, 2007, 2008]. In a recent study, a HIR subgroup was reported to constitute $47 \%$ of all cryptorchid boys [Bilius et al., 2015].

Microarray analyses of differentially expressed genes in the HIR group, compared to a low infertility risk group (LIR), revealed absent or low expression of multiple genes involved in the control of the HPG axis, i.e., DLX5, FGFR3, and ISL1 [Hadziselimovic et al., 2009, 2011]. Noticeably, insufficient EGR4 gene expression as well as a relative EGR1 deficiency was observed in the HIR group of cryptorchid boys [Hadziselimovic et al., 2009]. To date, the available molecular data favors LH deficiency, with EGR4 as a master regulator responsible for defective mini-puberty in the HIR group of cryptorchid boys.

Furthermore, in the serum of male Egr1-deficient mice, LH levels are found to be adequate in terms of the maintenance of Leydig cell steroidogenesis and fertility because of a partial functional redundancy with the closely related transcription factor Egr4 [Tourtellotte et al., 2000]. Moreover, a normal distribution of GnRH-containing neurons and normal innervation of the median eminence in the hypothalamus, as well as decreased levels of LH gene expression in Egr4/Egr1- versus Egr1-deficient male mice, indicates a defect of $\mathrm{LH}$ regulation. These results emphasize a novel level of functional redundancy between Egr4 and Egr1 in regulating LH production in male mice [Tourtellotte et al., 2000].

The aim of this work was to validate and extend our previous microarray study by analyzing different groups of cryptorchid boys with identical histological changes. The RNA-seq data has uncovered additional deregulated genes in the HIR group, with potential involvement in the HPG axis.

\section{Materials and Methods}

Study Population and Biopsy Sample Collection

We selected 15 patients with isolated cryptorchidism based on histological results and divided them into 2 groups. Seven belonged to the HIR and 8 to the LIR group. The cryptorchid boys (7 unilateral and 8 bilateral undescended testes) had a median age of 15 months (range 7-55 months) and had no prior hormonal or surgical treatment. Cryptorchid testis is defined as a testis localized outside the scrotum and incapable of being brought into a stable scrotal position. All undescended testes in this study were located in the inguinal region. The cryptorchid boys entering the study underwent an extensive examination with no clinical signs of developmental malformations or syndromes. Performing clinical examination in accordance with STROBE criteria for case-controlled studies [von Elm et al., 2014], we excluded small testes, small penis, lack of normal scrotal rugae and pigmentation and gynecomastia. We further determined serum FSH, LH, testosterone, and inhibin levels [Verkauskas et al., 2016]. No MRI scans of the brain and sella turcica were performed. No clinical symptoms were found for hyperprolactinemia, pituitary lesions (tumor, granuloma, and abscess), Cushing syndrome, severe or chronic illness, trauma or surgery, and genetic mutations such as Prader-Willi syndrome. None of our patients suffered from a systemic diseases such as hemochromatosis, sarcoidosis, and histiocytosis X.

Testicular biopsies were taken at the time of orchidopexy. A 5 -mm incision was made into the tunica albuginea in the superior pole of the testis, and a sample of approximately the size of a rice grain was isolated from the protruding tissue using separate blades. The sample was then divided into 2 small fragments, one of which was fixed in glutaraldehyde for histological processing. The second 
was immediately immersed in RNAlater ${ }^{\complement}$ (ThermoFisher Scientific) and stored at $-80^{\circ} \mathrm{C}$ until further processing for RNA extraction and RNA sequencing.

Histological Analyses

Biopsies were fixed in 3\% glutaraldehyde in PBS and were then embedded in Epon resin. Semi-thin sections $(1 \mu \mathrm{m})$ were cut on a Reichert Om-U3 ultramicrotome, mounted on glass slides, stained with $1 \%$ toluidine blue, and examined under a light microscope at a total magnification of $600 \times$. Biopsy material from all patients was histologically examined by 2 authors (F.H., D.D.), experienced in analyzing semi-thin sections of prepubertal testes.

During histological analyses, biopsies were evaluated with regard to their number of spermatogonia per tubule (S/T) and presence of dark-type (Ad) spermatogonia. In the prepubertal testis, Ad spermatogonia are identified according to criteria first published by Seguchi and Hadziselimovic [1974]. This type of germ cell has a typical halo in the nucleus, termed the rarefication zone, and a cytoplasm with a darker aspect in comparison to Ap or fetal spermatogonia [Seguchi and Hadziselimovic, 1974]. Crucially, the predominant factor in the development of infertility is the observation that gonadotropin levels show a more striking correlation with the presence or absence of Ad spermatogonia in both gonads than with the category of undescended testes, i.e., unilateral or bilateral [Hadziselimovic and Hoecht, 2008]. For each biopsy, at least 100 tubular cross sections were evaluated. On the basis of this evaluation, biopsies were categorized into 2 groups, HIR and LIR, respectively, of infertility outcome. The HIR group included biopsies with $\mathrm{S} / \mathrm{T} \leq 0.2$ and no Ad and the LIR group recorded S/T scores of $>0.6$ with Ad. Biopsies categorized in the HIR $(\mathrm{n}=7)$ and LIR $(\mathrm{n}=8)$ groups were included in our RNA-seq analyses.

\section{RNA Isolation and Purification}

Total RNA was isolated from RNAlater ${ }^{\complement}$-treated biopsies. Briefly, tissues were mixed with $500 \mu \mathrm{l}$ of Tri-Reagent (Ambion) and homogenized for $2 \mathrm{~min}$ at $30 \mathrm{~Hz}$ using a TissueLyser II (Qiagen). Following homogenization, $500 \mu \mathrm{l}$ of absolute ethanol was added to each sample and mixed. The mixture was loaded onto Zymo-Spin IIC columns, and RNA was further purified using a Direct-Zol kit (ZymoResearch) according to the manufacturer's instructions. These included DNAse I treatment to remove any contaminating genomic DNA. The concentration of the eluted RNA was determined using a NanoDrop 2000 spectrophotometer (ThermoScientific) and quality-controlled using RNA 6000 Pico chips on a 2100 BioAnalyzer (Agilent).

\section{RNA Library Preparation}

Library preparation was performed from 300 ng total RNA using the TruSeq Stranded Total RNA Kit with Ribo-Zero Gold (Illumina). This workflow includes a prior step to remove ribosomal RNA in order to facilitate sequencing of both coding and noncoding RNAs and the ligation of specific indexes. Libraries were quality-controlled on the Fragment Analyzer (Advanced Analytical) using the Standard Sensitivity NGS Fragment Analysis Kit (Advanced Analytical). Libraries were of excellent quality with an average concentration of $128 \mathrm{nmol} / \mathrm{l}$ and average size of $367 \mathrm{bp}$. Samples were pooled to equivalent molarity in 2 equally large series of samples. Each pool was run on the Fragment Analyzer for quality control purposes and quantification.

\section{RNA Sequencing}

Library pools were adjusted to 1.3 or $1.4 \mathrm{pM}$ and used for clustering on the NextSeq 500 instrument (Illumina). Each of the 2 pools was sequenced on a 2 flow-cell to yield more reads, increasing our ability to detect poorly expressed transcripts and noncoding RNAs. Samples were sequenced as singlereads of 81 bases using the NextSeq 500/550 High Output Kit v2 kit 75-cycles (Illumina). The pools yielded between 91 and $95 \%$ of pass-filter reads equivalent to, or in excess of, the Illumina quality score of 30. Primary data analyses were performed with the Illumina RTA version 2.4.6 and bcl2fastq v2.17.1.14.

\section{RNA-Seq Data Analyses}

Reads were aligned to the UCSC human hg19 genome assembly (GRCh37) using STAR (version 2.5.0a) with parameters that report no more than a single alignment per read for reads with up to 20 hits. Around 60 million reads were mapped per sample (range from 46 to 100 million reads). On average, 96\% of reads were mapped (range from 94 to $97.5 \%$ ) with less than $10 \%$ of multimappers.

To identify any potentially contaminating ribosomal RNA or nucleic acids from other species, a random subset of reads from each sample was also aligned to a collection of alternative references. Apart from a few reads of bacterial origin (typically E. coli, $\sim 1 \%$ ), only nonribosomal human sequences were detected in all samples. Raw data files are available at the Database of Genotypes and Phenotypes (dbGaP).

\section{Expression Level Quantification}

To quantify gene expression, alignments were counted per gene and sample using the QuasR Bioconductor package [Gaidatzis et al., 2015] with known genes from the UCSC knownGene table (TxDb.Hsapiens.UCSC.hg19.knownGene Bioconductor package from 2015-10-07), combined with noncoding RNAs from the UCSC lincRNAsTranscripts table (TxDb.Hsapiens. UCSC.hg19.lincRNAsTranscripts Bioconductor package from 2015-10-07).

To remove technical biases and enable a comparison of transcripts, the resulting raw data (mapped read counts per gene) were normalized for RNA length and for total read number in the lane to reads per $\mathrm{kb}$ and per million mapped reads (RPKM).

\section{Data and Differential Gene Expression Analyses}

Normalized data for all samples were analyzed by unsupervised principal component analysis (PCA) and hierarchical cluster analysis (HCA) to identify patterns in the dataset and highlight similarities and differences among the samples. The effect of patient age as a potential confounder was tested but was found to be insignificant for our statistical analyses. Age did not significantly associate with patient group (one-way ANOVA, p value $=0.349$ ), and analyses of differential expression related to age failed to yield any significant genes (false discovery rate, FDR $<0.05$ ) with a $>2$-fold expression difference over 6 months. Data analyses were therefore performed to determine genes differentially expressed in HIR versus LIR patients, excluding age as a factor in $\mathrm{R}$ using the edgeR package [Robinson et al., 2010] and the therein implemented quasi-likelihood methods of Lund et al. [2012]. Only genes with at least 1 read per million in at least 2 samples were included. Differentially expressed genes were defined as genes displaying a FDR $<0.05$ and an absolute change in expression of at least 2 -fold. 


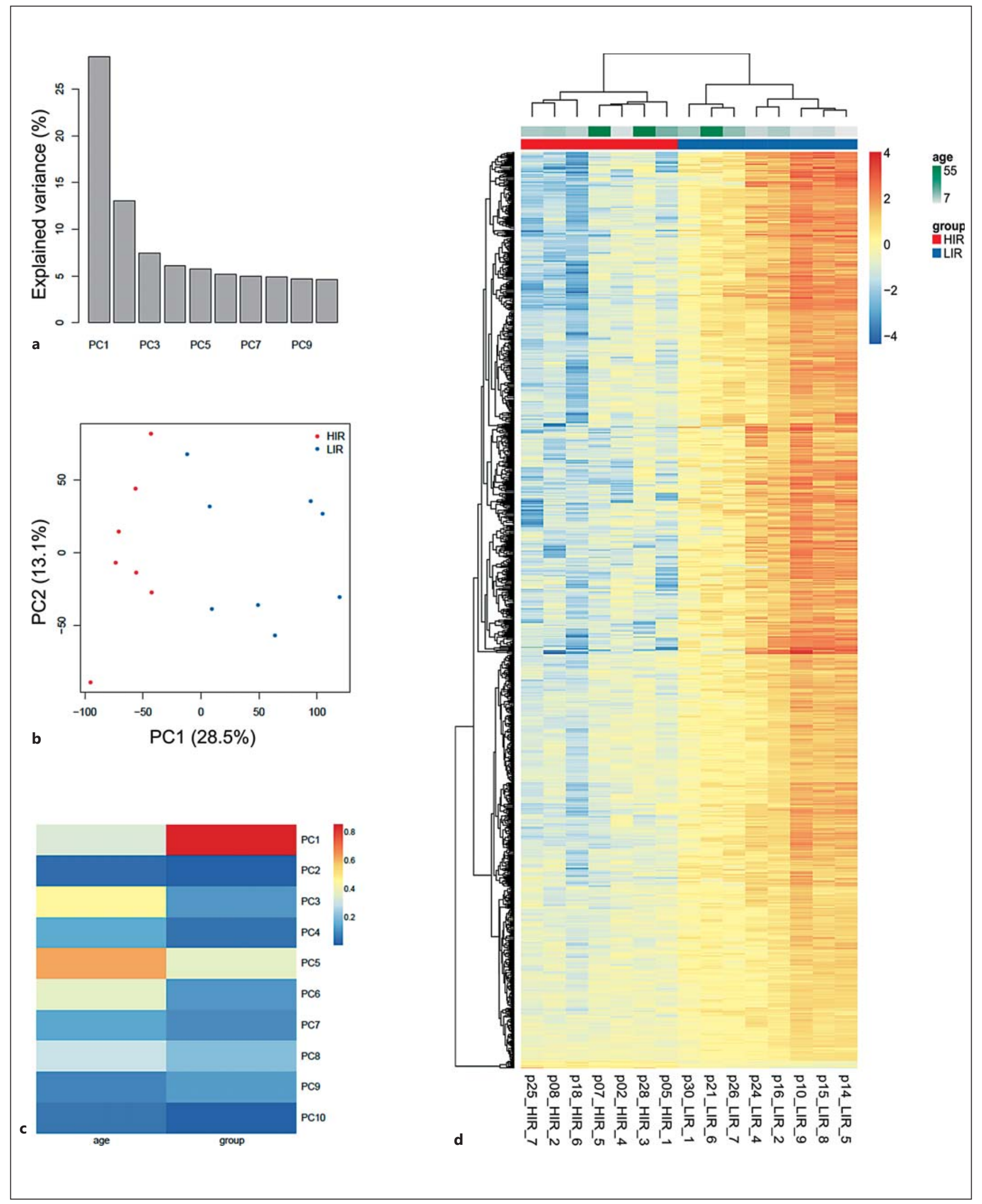

(For legend see next page.) 


\section{Results}

Illumina sequencing and gene expression quantification of barcoded libraries from 7 HIR and 8 LIR testes yielded a total of 23,737 detected genes (out of a total of 45,089 quantified genes). Global characterization of the dataset using PCA showed that the major source of variance $(28.5 \%$ of the total variance in the dataset) corresponded to the differences between HIR and LIR patient groups (fig. 1a, b). Other experimental factors had lower contribution to the variance, for example patient age contributed to principle components 3 and 5 (fig. 1c). Differential gene expression analysis identified 2,033 significant genes with a FDR $<0.05$ and an absolute change of at least 2 -fold between the HIR and LIR groups. Most of these 2,033 differential genes were expressed at a lower level in the HIR group $(1,986,97.7 \%)$, while only 47 (2.3\%) were upregulated in the HIR versus the LIR group.

A hierarchical clustering of relative gene expression levels for the top 1,000 significant genes identified in the HIR and LIR biopsies is represented as a heatmap in figure $1 \mathrm{~d}$. Distinct gene expression profiles in terms of their categorization in either the HIR or LIR groups could be clearly identified.

\section{RNA-Seq Confirmation of Genes Previously Reported} to Be Downregulated in the HIR Group

An independent study analyzing differentially expressed genes involved in germ cell development and in the HPG axis (using oligonucleotide microarray technology) reported 59 downregulated genes in the HIR versus the LIR group [Hadziselimovic et al., 2011]. The differential gene expression analyses conducted in this study confirmed 57 of these gene targets (table 1). All confirmed differentially expressed genes were significant with a FDR $<0.05$ and an absolute fold-change of at least 2 . The 3 exceptions (DDX25, NRG1, and TLE1) were also significant but had absolute fold-changes $<2$. The 2 genes $D A Z 1$ and $T D R D 10$, which were previously reported to be significantly downregulated in HIR compared to a control group but not compared to a LIR group, were identified in this study to be significantly downregulated in HIR versus LIR. In contrast, we could not confirm a differential expression for the genes MBD2, SRY, or UTF1. Additionally, while $R B M Y 1 A 1$ was not assessed in this study, we did identify a decreased expression of the members B, $\mathrm{E}, \mathrm{F}$, and $\mathrm{J}$ of the same RBMY gene family 1 .

\section{Identification of PROK2 and Other Novel Genes That Are Downregulated in HIR, with Potential Involvement in the HPG Axis and Pituitary Development}

We next screened for additional, as yet unknown genes that play a role in the HPG axis and for which mRNA levels were also decreased in the HIR group. We identified 38 additional genes, including prokineticin 2 (PROK2), early growth response 2 (EGR2), paired-like homeodomain 1 (PITX1), chromo domain helicase DNA binding
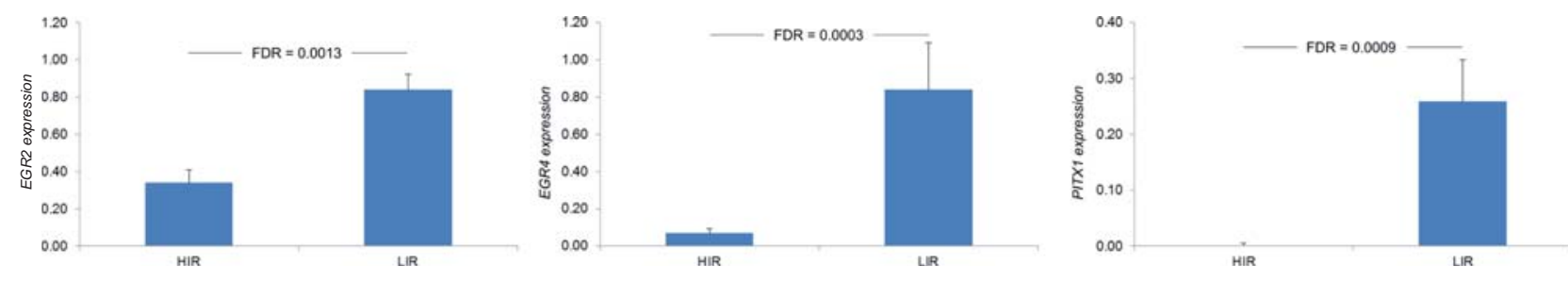

Fig. 2. Expression levels of EGR2, EGR4, and PITX1 analyzed by RNA sequencing in HIR and LIR cryptorchid testes. Gene expression shown as median RPKM values, mean absolute deviations and FDR values is presented.

Fig. 1. Principal component analysis and relative gene expression of HIR and LIR patients. a Percent of total variance explained by the first 10 principle components (PCs). PC1 accounts to $28.5 \%$ of the total variance. b Projection of individual samples using PC1 and PC2. Percent of the explained variance is shown in parentheses. c Heatmap with Pearson's correlation coefficients between PCs and patient age or group (HIR or LIR). PC1 is strongly associated with patient group, whereas PC5 is weekly associated with age. d Heatmap showing the $\log 2$ expression of the 1,000 most significant differential genes between HIR and LIR patients relative to the average expression of each gene across all patients. Patient age and group are indicated by color on top of the heatmap, and both patients and genes have been reordered by hierarchical clustering to group similar profiles (clustering dendrograms shown on top and on the left side). 
Table 1. Genes involved in germ cell development and in the HPG axis (bold) and downregulated in the HIR group

\begin{tabular}{|c|c|c|c|c|}
\hline Gene ID & Name & $\log \mathrm{FC}^{\mathrm{a}}$ & FDR & pHAZR/LAZR \\
\hline ALDH1A2 & aldehyde dehydrogenase 1 family, member A2 & -2.36946 & 0.000175 & 0.0006 \\
\hline AMPH & amphiphysin & -1.51873 & 0.000888 & 0.02 \\
\hline$C B L$ & Cbl proto-oncogene, E3 ubiquitin protein ligase & -1.28185 & 0.0007 & 0.0004 \\
\hline CDC20 & cell division cycle 20 & -2.5884 & 0.000154 & 0.003 \\
\hline CLGN & calmegin & -1.04018 & 0.014042 & 0.01 \\
\hline CSRP2 & cysteine and glycine-rich protein 2 & -1.79674 & 0.000264 & 0.002 \\
\hline$D A Z L$ & deleted in azoospermia-like & -1.30306 & 0.007261 & 0.03 \\
\hline DDX25 & DEAD (Asp-Glu-Ala-Asp) box helicase 25 & -0.97862 & 0.003528 & 0.001 \\
\hline DDX4 & DEAD (Asp-Glu-Ala-Asp) box polypeptide 4 & -2.86159 & 0.000222 & $8.25 \mathrm{E}-05$ \\
\hline DMRTB1 & DMRT-like family B with proline-rich C-terminal, 1 & -3.26495 & 0.000154 & 0.003 \\
\hline DPYSL4 & dihydropyrimidinase-like 4 & -1.09804 & 0.02908 & 0.001 \\
\hline DTL & denticleless E3 ubiquitin protein ligase homolog (Drosophila) & -1.51525 & 0.00036 & 0.0007 \\
\hline DUSP5 & dual specificity phosphatase 5 & -2.14862 & 0.000267 & 0.008 \\
\hline FST & follistatin & -1.99538 & 0.000624 & 0.001 \\
\hline GAGE1 & G antigen 1 & -1.79238 & 0.007285 & 0.001 \\
\hline GTSF1 & gametocyte-specific factor 1 & -2.96989 & 0.000154 & 0.0001 \\
\hline ID4 & inhibitor of DNA binding 4, dominant negative helix-loop-helix protein & -1.53417 & 0.001103 & 0.0002 \\
\hline ISL1 & ISL LIM homeobox 1 & -2.09731 & 0.000515 & 0.0004 \\
\hline KIF21B & kinesin family member $21 \mathrm{~B}$ & -1.9747 & 0.000218 & 0.0001 \\
\hline LIN28B & lin-28 homolog B (C. elegans) & -2.2908 & 0.000264 & 0.01 \\
\hline$L I N 7 B$ & lin-7 homolog B (C. elegans) & -1.94848 & 0.000322 & 0.02 \\
\hline MAGEA4 & melanoma antigen family A4 & -2.65916 & 0.000218 & 0.009 \\
\hline MAGEC1 & melanoma antigen family C1 & -2.50237 & 0.000154 & 8.24E-05 \\
\hline MND1 & meiotic nuclear divisions 1 homolog (S. cerevisiae) & -2.18184 & 0.000547 & 0.004 \\
\hline$M O R C 1$ & MORC family CW-type zinc finger 1 & -2.46752 & 0.000281 & 0.0001 \\
\hline NLRP2 & NLR family, pyrin domain containing 2 & -1.4955 & 0.001143 & 0.005 \\
\hline NMU & neuromedin U & -2.1983 & 0.001858 & 0.005 \\
\hline NRG1 & neuregulin 1 & -0.92134 & 0.013631 & 0.004 \\
\hline SSX2 & synovial sarcoma, X breakpoint 2 & -2.02297 & 0.001142 & 0.003 \\
\hline$S Y C P 3$ & synaptonemal complex protein 3 & -2.24491 & 0.002046 & 0.0002 \\
\hline TAF5 & TAF5 RNA polymerase II, TATA box binding protein (TBP)-associated factor, $100 \mathrm{kDa}$ & -1.08538 & 0.000894 & 0.005 \\
\hline TAF7L & TAF7-like RNA polymerase II, TATA box binding protein (TBP)-associated factor, $50 \mathrm{kDa}$ & -1.98595 & 0.001673 & 0.03 \\
\hline TDRD10 & tudor domain containing 10 & -1.80786 & 0.000981 & n.s. \\
\hline TDRD5 & tudor domain containing 5 & -2.64116 & 0.000294 & 0.01 \\
\hline TDRD 6 & tudor domain containing 6 & -1.80614 & 0.000154 & 0.0003 \\
\hline TDRD9 & tudor domain containing 9 & -1.52239 & 0.00057 & 0.009 \\
\hline TEX14 & testis expressed 14 & -1.53753 & 0.000453 & 0.001 \\
\hline TLE1 & transducin-like enhancer of split 1 (E(sp1) homolog, Drosophila) & -0.90022 & 0.001061 & 0.0001 \\
\hline TSPY1 & testis-specific protein, Y-linked 1 & -2.49393 & 0.000323 & 8.24E-05 \\
\hline WNT3 & wingless-type MMTV integration site family, member 3 & -2.64239 & 0.000154 & 0.0003 \\
\hline MBD2 & methyl-CpG binding domain protein 2 & n.s. & n.s. & 0.03 \\
\hline$S R Y$ & sex-determining region $\mathrm{Y}$ & n.s. & n.s. & 0.002 \\
\hline UTF1 & undifferentiated embryonic cell transcription factor 1 & n.s. & n.s. & 0.0002 \\
\hline RBMY1A1 & RNA binding motif protein, Y-linked, family 1 , member A1 & NA & NA & 0.0008 \\
\hline$R B M Y 1 B$ & RNA binding motif protein, Y-linked, family 1 , member B & -1.93256 & 0.000405 & \\
\hline RBMY1E & RNA binding motif protein, Y-linked, family 1 , member $\mathrm{E}$ & -1.90322 & 0.002026 & \\
\hline$R B M Y 1 F$ & RNA binding motif protein, Y-linked, family 1 , member $F$ & -2.02817 & 0.000822 & \\
\hline RBMY1J & RNA binding motif protein, Y-linked, family 1 , member J & -1.95224 & 0.000653 & \\
\hline
\end{tabular}

Table adapted from Hadziselimovic et al. [2009].

a Absolute fold-changes $<2$ are highlighted in italics.

Gene Expression in Cryptorchidism with Defective Mini-Puberty 


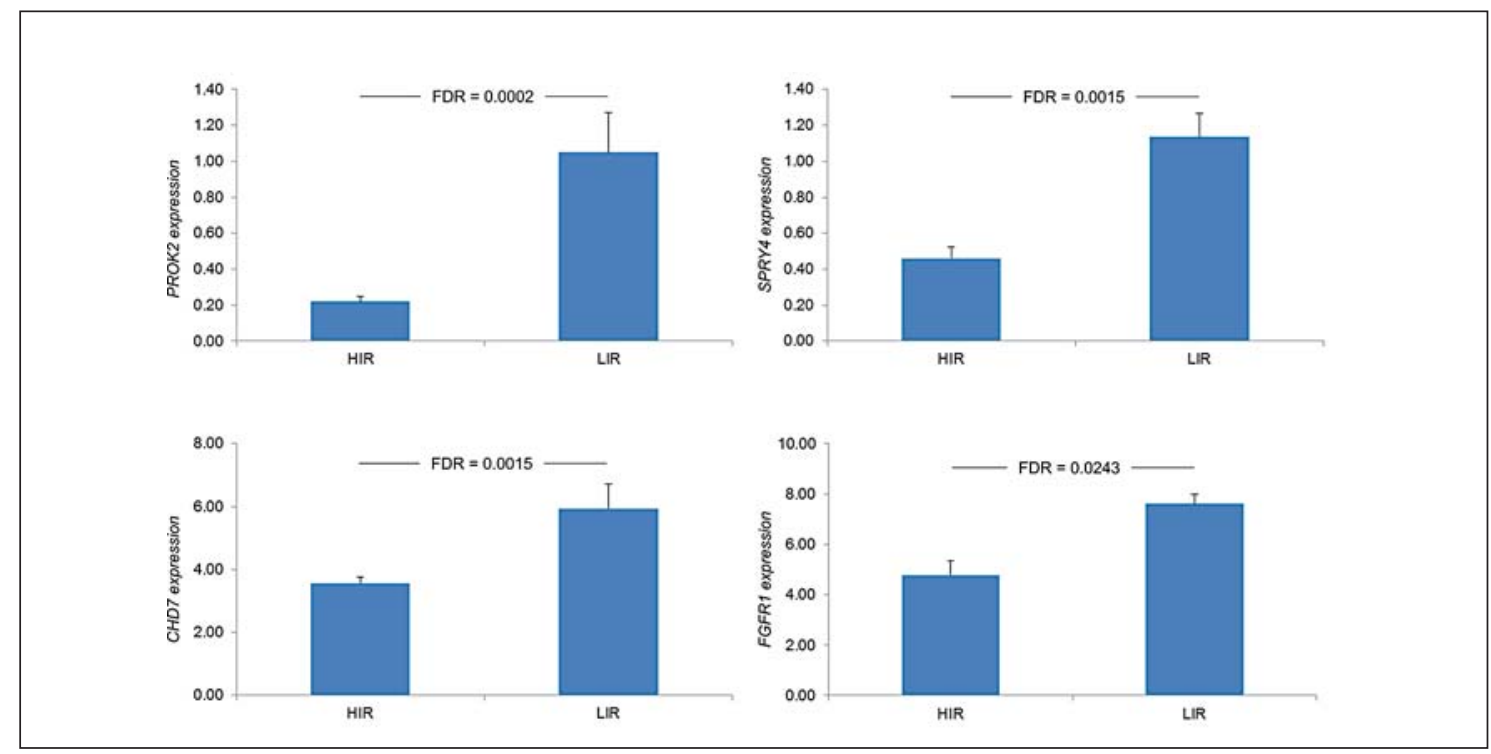

Fig. 3. Expression levels of Kallmann syndrome involved genes PROK2, SPRY4, CHD7 and FGFR1 analyzed by RNA sequencing in HIR and LIR cryptorchid testes. Gene expression shown as median RPKM values, mean absolute deviations and FDR values is presented.

protein 7 (CHD7), and sprout homolog 4 (SPRY4) as well as fibroblast growth factor receptor 1 (figs. 2, 3; table 2). With the exception of 7 genes (ATF3, CHD7, CLOCK, FGFR1, FGFRL1, RUNX1, and SEMA4D), all were expressed with an absolute fold-change $>2$.

Furthermore, OTX2, PITX1, PITX2, GATA2, LHX2, $L H X 6$, and $L H X 8$ transcripts involved in pituitary development and differentiation had a lower signal in the HIR group (tables 1, 2). ISL1, a paralog of LHX4, showed a significantly lower signal in HIR (table 1). As a paralog, this gene is related to LHX4 by duplication within the genome and subsequently evolved a new function. The LHX4 gene encodes a member of a large protein family which contains the LIM domain, a unique cysteine-rich zinc-binding domain. The encoded protein is a transcription factor involved in the control of differentiation and development of the pituitary gland.

\section{Discussion}

GeneChip-based whole genome expression profiling of testicular tissue collected from cryptorchid boys with impaired mini-puberty revealed a decreased expression of multiple genes essential for HPG axis function. RNAseq analysis of a new group of HIR and LIR patients that we selected using identical histological criteria confirmed and extended our previous results [Hadziselimovic et al., $2009,2011]$. Nearly all of the genes implicated in the HPG axis that were reported to be downregulated in the HIR group were confirmed by our RNA-seq analysis (table 1). In addition, we identified genes for which transcripts are decreased in HIR that are also known to be involved in the HPG axis (figs. 2, 3; table 2). The reproducibility of the gene sets downregulated in either study of cryptorchid boys (with defective mini-puberty and lack of Ad spermatogonia) clearly supports a role for the HPG axis in the development of azoospermia and infertility.

Moreover, we confirmed in HIR patients a previously reported strongly reduced expression of EGR4 and found a significant relative downregulation of EGR2 and PITX1, (fig. 2; table 1). Although a critical role of EGR2 has been shown in hindbrain development [Schneider-Maunoury et al., 1993; Swiatek and Gridley, 1993] and peripheral nerve myelination [Topilko et al., 1994], no specific function in testis or spermatogenesis has been described yet. In contrast, its family member EGR4 is expressed within maturing germ cells and plays a critical role in spermatogenesis [Tourtellotte et al., 1999]. Furthermore, it was suggested that gene misregulation in Leydig cells (mediated by Egr1) and in germ cells (mediated by Egr4) disrupts critical intrinsic regulatory pathways leading to complete spermatogenic arrest in the absence of both transcription factors [Tourtellotte et al., 2000]. A specif- 
Table 2. New identified genes downregulated in HIR and involved in the HPG axis

\begin{tabular}{|c|c|c|c|c|c|}
\hline Gene ID & Name & HIR median & LIR median & $\log \mathrm{FC}^{\mathrm{a}}$ & FDR \\
\hline$A R I D 3 B$ & AT rich interactive domain 3B (BRIGHT-like) & 1.25 & 3.35 & -1.44965 & 0.000171 \\
\hline ATF3 & activating transcription factor 3 & 0.47 & 1.10 & -0.96099 & 0.009544 \\
\hline AXIN2 & axin 2 & 2.33 & 5.75 & -1.28981 & 0.000283 \\
\hline$B M P 7$ & bone morphogenetic protein 7 & 0.27 & 0.76 & -1.58963 & 0.00144 \\
\hline$B M P R 1 B$ & bone morphogenetic protein receptor, type IB & 0.72 & 3.90 & -2.5653 & 0.000184 \\
\hline CHD7 & chromodomain helicase DNA binding protein 7 & 3.57 & 5.94 & -0.72742 & 0.001522 \\
\hline CLOCK & clock circadian regulator & 9.47 & 13.82 & -0.46419 & 0.01024 \\
\hline$D L X 2$ & distal-less homeobox 2 & 0.05 & 0.28 & -2.60142 & 0.003894 \\
\hline$D L X 3$ & distal-less homeobox 3 & 0.06 & 0.28 & -2.07726 & 0.006612 \\
\hline$D L X 5$ & distal-less homeobox 5 & 0.20 & 0.87 & -1.92109 & 0.00407 \\
\hline DUSP4 & dual specificity phosphatase 4 & 0.21 & 0.74 & -1.73444 & 0.000154 \\
\hline EBF3 & early B-cell factor 3 & 0.33 & 1.59 & -1.94656 & 0.000218 \\
\hline EGR2 & early growth response 2 & 0.34 & 0.84 & -1.17858 & 0.001333 \\
\hline FGF14 & fibroblast growth factor 14 & 0.19 & 0.49 & -1.23942 & 0.020814 \\
\hline FGFR1 & fibroblast growth factor receptor 1 & 4.79 & 7.61 & -0.49372 & 0.024252 \\
\hline FGFR2 & fibroblast growth factor receptor 2 & 1.98 & 4.32 & -1.25292 & 0.00052 \\
\hline FGFRL1 & fibroblast growth factor receptor-like 1 & 1.33 & 2.84 & -0.97919 & 0.010591 \\
\hline GATA2 & GATA binding protein 2 & 0.33 & 1.06 & -1.52714 & 0.000339 \\
\hline ISL2 & ISL LIM homeobox 2 & 0.08 & 0.27 & -1.6183 & 0.008855 \\
\hline KLF4 & Kruppel-like factor 4 (gut) & 1.43 & 4.11 & -1.16046 & 0.008282 \\
\hline LHX2 & LIM homeobox 2 & 0.03 & 0.28 & -3.13022 & 0.0019 \\
\hline LHX6 & LIM homeobox 6 & 0.35 & 1.17 & -1.72084 & 0.000238 \\
\hline LIN28B & lin-28 homolog B (C. elegans) & 0.43 & 1.78 & -2.2908 & 0.000264 \\
\hline MSX1 & msh homeobox 1 & 0.29 & 0.69 & -1.37378 & 0.00227 \\
\hline NHLH2 & nescient helix loop helix 2 & 0.21 & 0.62 & -1.65737 & 0.000772 \\
\hline NOS1 & nitric oxide synthase 1 (neuronal) & 0.12 & 0.29 & -1.57741 & 0.000352 \\
\hline NR4A1 & nuclear receptor subfamily 4 , group A, member 1 & 1.52 & 2.80 & -1.05698 & 0.00366 \\
\hline OTX2 & orthodenticle homeobox 2 & 0.12 & 0.34 & -1.7389 & 0.03221 \\
\hline PCSK1N & proprotein convertase subtilisin/kexin type 1 inhibitor & 0.28 & 1.87 & -2.57264 & 0.003657 \\
\hline PITX1 & paired-like homeodomain 1 & 0.00 & 0.26 & -4.21104 & 0.000866 \\
\hline PITX2 & paired-like homeodomain 2 & 0.07 & 0.47 & -2.36513 & 0.00097 \\
\hline POU3F1 & POU class 3 homeobox 1 & 0.09 & 0.43 & -2.53507 & 0.000391 \\
\hline PROK2 & prokineticin 2 & 0.22 & 1.05 & -2.43563 & 0.000184 \\
\hline RUNX1 & runt-related transcription factor 1 & 0.40 & 0.67 & -0.95564 & 0.008482 \\
\hline SEMA3F & $\begin{array}{l}\text { sema domain, immunoglobulin domain (Ig), short basic domain, secreted, } \\
\text { (semaphorin) } 3 \mathrm{~F}\end{array}$ & 0.15 & 0.43 & -1.67332 & 0.004377 \\
\hline SEMA4D & $\begin{array}{l}\text { sema domain, immunoglobulin domain (Ig), transmembrane domain (TM) } \\
\text { and short cytoplasmic domain, (semaphorin) 4D }\end{array}$ & 2.27 & 4.07 & -0.83474 & 0.001076 \\
\hline SPRY4 & sprouty homolog 4 (Drosophila) & 0.46 & 1.14 & -1.05327 & 0.001517 \\
\hline TRPC3 & transient receptor potential cation channel, subfamily C, member 3 & 0.45 & 1.04 & -1.34278 & 0.004737 \\
\hline
\end{tabular}

a Absolute fold-changes $<2$ are highlighted in italics.

ic function of PITX1 and PITX2 in the testis is yet unknown. However, all 4 genes, EGR4, EGR2, PITX1, and PITX2, are directly involved in the regulation and expression of the LH $\beta$ subunit in the pituitary gland [Kaiser et al., 1998, 2000; Wei et al., 2002; Fortin et al., 2009]. Noticeably, we identified a decreased expression of the genes $M S X 1, D L X 2, D L X 3, N R 4 A 1$, and $L H X 3$ in the HIR group (table 2). In mice, Msx1 functions as a repressor and Dlx2 and Dlx3 as transcriptional activators of glycoprotein hormone a subunit ( $\alpha$ GSU) and GnRH receptor (GnRHR) [Xie et al., 2013]. NR4A1 and LHX3 have been implicated in GnRHR gene expression [Sadie et al., 2003; McGillivray et al., 2005]. Thus, our finding of downregulated genes directly involved in the modulation of $\mathrm{LH} \beta$, aGSU, and GnRHR expression implies an effect on LH production and provides a plausible explanation for the reduced LH levels measured in HIR patients.

Kallmann syndrome accounts for $30-40 \%$ of the total caseload of ICH and has been considered to be a distinct subgroup of ICH [Bonomi et al., 2012]. Both Kallmann syndrome and HIR group cryptorchid patients have the following in common: (a) low LH levels, (b) extensive Leydig cell atrophy and impaired testosterone secretion, (c) similar testicular histology lacking gonocytes transformation into spermatogonial stem cells, (d) infertility frequently combined with azoospermia, and (e) importantly, both groups can be successfully treated with $\mathrm{GnRH}$ ( $\mathrm{LH}$ releasing hormone) to rescue fertility [Cole et al., 2008; Sinisi et al., 2008]. In contrast to Kallmann syndrome, which is caused by one or several mutations in 22 
genes [Valdes-Socin et al., 2014; Kim, 2015], the HIR group showed 4 differentially expressed genes: FGFR1, PROK2, CHD7 and SPRY4 which are important for $\mathrm{LH}$ secretion (fig. 3). Thus, observed LH insufficiency in the HIR group is likely to result from a compromised gene expression. Furthermore, in contrast to boys with Kallmann syndrome, cryptorchid boys in the HIR group had normal FSH and inhibin plasma values [Verkauskas et al., 2016]. Our data do not show a direct causal relationship since they are not confirmed experimentally. However, observed insufficient gene expression in the HIR but not in the LIR group strongly supports the notion that the above described 5 main points of similarities between Kallmann syndrome and the HIR group are caused by an abnormal gene expression. This is the first step in understanding a potential new risk factor for infertility development in cryptorchidism, which is vital if we are to develop new approaches for hormonal treatment and early risk detection in the future.

Consequently, in cases of isolated congenital cryptorchidism, we hypothesize that oligogenicity contributes to the phenotypic presentation of defective mini-puberty and infertility. This observation indicates a broader genetic spectrum of ICH and may explain the development of defective mini-puberty in otherwise healthy cryptorchid boys. Interestingly, among other tissues PROK2 expression was found predominantly in brain and testis [Wechselberger et al., 1999]. In human and mice, the highest similarity in gene expression profiles is between brain and testes [Guo et al., 2003]. Besides its role in the HPG axis, PROK2 is highly expressed in primary spermatocytes and in seminiferous tubules in the testis [Wechselberger et al., 1999; Samson et al., 2004]. Thereby, PROK2 functions as an angiogenic mitogen to promote growth and survival of the testicular interstitial vessels [LeCouter et al., 2003]. Subsequently, this might affect the modulation of testosterone secretion out of the testis and of regulatory factors into the testis [Samson et al., 2004]. In addition, PROK2 functions as a mediator of the inflammatory response [Samson et al., 2004] and may perform this function in the context of the epididymis [LeCouter et al., 2003]. Therefore, the presence of PROK2 transcripts in the primary spermatocytes associated with an angiogenic role in the testis suggests an important role of this prokineticin in spermatogenesis.

As a consequence of impaired mini-puberty, $97 \%$ of cryptorchid HIR males were infertile with an average of $9.1 \times 10^{6}$ sperm per ejaculate, while $33 \%$ out of this group developed azoospermia [Hadziselimovic, 2008; Hadziselimovic et al., 2011]. However, if cryptorchid boys with impaired mini-puberty received treatment with a $\mathrm{GnRH}$ analogue following orchidopexy, a normal spermiogram was achieved in $86 \%$ of subjects [Hadziselimovic, 2008]. Thus, hormonal treatment with GnRH in early childhood permanently restored fertility and prevented the development of azoospermia [Hadziselimovic, 2008]. Superior fertility results were achieved for hormone (Buserelin)treated cryptorchid boys $<6$ years of age [Hadziselimovic and Herzog, 1997; Hadziselimovic, 2008]. Therefore, it seems likely that the observed success with Buserelin depends on an optimal treatment window when a permanent reversal of PROK2 neuronal plasticity can be achieved.

In conclusion, the results obtained from RNA-seq analyses strongly support the theory that an impaired mini-puberty causes azoospermia and infertility in cryptorchidism. Multiple differences in gene expression identified when comparing the HIR and LIR groups confirmed the results of our previously published whole genome microarray expression analyses and further underscore the importance of an intact HPG axis in fertility development and spermatogenesis. Thus, molecular observations support a crucial role for PROK2 in the physiology of mini-puberty with impaired PROK2/CHD7/ FGFR1/SPRY4 gene expression inducing LH deficiency as controlled by the regulators EGR4 and PITX1.

\section{Acknowledgments}

This study was supported in part by the European Social Fund under the Global Grant measure. Work in the Stadler group is supported by funding from the MetastasiX project of SystemsX.ch. We thank Manuel Kohler, Department of Biosystems Science and Engineering (D-BSSE), ETH Zurich for helping us perform RNA sequencing.

\section{Statement of Ethics}

In accordance with the Declaration of Helsinki, the Institutional Review Board and the Independent Ethics Committee of Vilnius University approved all aspects of this study. Treatment protocols and approval for testicular biopsies were obtained following the guidelines from the Ethics Committee of the University of Vilnius. Approval was also provided for research involving the use of material (data records or biopsy specimens) that had been collected for non-research purposes (Vilnius Regional Biomedical Research Ethics Committee, No. 158200-580-PPI-17).

\section{Disclosure Statement}

The authors have no conflicts of interest to disclose. 


\section{References}

-Antunes JL, Carmel PW, Housepian EM, Ferin $\mathrm{M}$ : Luteinizing hormone-releasing hormone in human pituitary blood. J Neurosurg 49: 382-386 (1978).

-Bilius V, Verkauskas G, Dasevicius D, Kazlauskas V, Malcius D, Hadziselimovic F: Incidence of high infertility risk among unilateral cryptorchid boys. Urol Int 95:142-145 (2015).

-Bonomi M, Libri DV, Guizzardi F, Guarducci E, Maiolo E, et al: New understandings of the genetic basis of isolated idiopathic central hypogonadism. Asian J Androl 14:49-56 (2012).

-Chung E, Brock GB: Cryptorchidism and its impact on male fertility: a state of art review of current literature. Can Urol Assoc J 5:210214 (2011).

-Cole LW, Sidis Y, Zhang C, Quinton R, Plummer $\mathrm{L}$, et al: Mutations in prokineticin 2 and prokineticin receptor 2 genes in human gonadotrophin-releasing hormone deficiency: molecular genetics and clinical spectrum. J Clin Endocrinol Metab 93:3551-3559 (2008).

-Corbier P, Edwards DA, Roffi J: The neonatal testosterone surge: a comparative study. Arch Int Physiol Biochim Biophys 100:127-131 (1992).

- Fedder J, Crüger D, Oestergaard B, Petersen GB: Etiology of azoospermia in 100 consecutive nonvasectomized men. Fertil Steril 82:14631465 (2004).

- Forest MG, Sizonenko PC, Cathiard AM, Bertrand J: Hypophyso-gonadal function in humans during the first year of life. Evidence for testicular activity in early infancy. J Clin Invest 53:819-828 (1974).

Fortin J, Lamba P, Wang Y, Bernard DJ: Conservation of mechanisms mediating gonadotrophin-releasing hormone 1 stimulation of human luteinizing hormone $\beta$ subunit transcription. Mol Hum Reprod 15:77-87 (2009).

-Gaidatzis D, Lerch A, Hahne F, Stadler MB: QuasR: quantification and annotation of short reads in R. Bioinformatics 31:11301132 (2015).

Guo J, Zhu P, Wu C, Yu L, Zhao S, Gu X: In silico analysis indicates a similar gene expression pattern between human brain and testis. Cytogenet Genome Res 103:58-62 (2003).

Hadziselimovic F: Successful treatment of unilateral cryptorchid boys risking infertility with LH-RH analogue. Int Braz J Urol 34:319-326 (2008).

-Hadziselimovic F, Herzog B: Treatment with a luteinizing hormone-releasing hormone analogue after successful orchiopexy markedly improves the chance of fertility later in life. J Urol 158:1193-1195 (1997)

-Hadziselimovic F, Hoecht B: Testicular histology related to fertility outcome and postpubertal hormone status in cryptorchidism. Klin Padiatr 220:302-307 (2008).
Hadziselimovic F, Emmons LR, Buser MW: A diminished postnatal surge of Ad spermatogonia in cryptorchid infants is additional evidence for hypogonadotropic hypogonadism. Swiss Med Wkly 134:381-384 (2004).

Hadziselimovic F, Zivkovic D, Bica DT, Emmons LR: The importance of mini-puberty for fertility in cryptorchidism. J Urol 174:15361539; discussion 1538-1539 (2005).

Hadziselimovic F, Höcht B, Herzog B, Buser MW: Infertility in cryptorchidism is linked to the stage of germ cell development at orchidopexy. Horm Res 68:46-52 (2007).

Hadziselimovic F, Hadziselimovic NO, Demougin P, Krey G, Hoecht B, Oakeley EJ: EGR4 is a master gene responsible for fertility in cryptorchidism. Sex Dev 3:253-263 (2009).

Hadziselimovic F, Hadziselimovic NO, Demougin P, Oakeley EJ: Testicular gene expression in cryptorchid boys at risk of azoospermia. Sex Dev 5:49-59 (2011).

-Hadziselimovic F, Hadziselimovic NO, Demougin P, Krey G, Oakeley EJ: Deficient expression of genes involved in the endogenous defense system against transposons in cryptorchid boys with impaired mini-puberty. Sex Dev 5:287-293 (2012).

Kaiser UB, Sabbagh E, Chen MT, Chin WW, Saunders BD: Sp1 binds to the rat luteinizing hormone beta (LHbeta) gene promoter and mediates gonadotropin-releasing hormonestimulated expression of the LHbeta subunit gene. J Biol Chem 273:12943-12951 (1998).

Kaiser UB, Halvorson LM, Chen MT: Sp1, steroidogenic factor 1 (SF-1), and early growth response protein 1 (egr-1) binding sites form a tripartite gonadotropin-releasing hormone response element in the rat luteinizing hormone-beta gene promoter: an integral role for SF-1. Mol Endocrinol 14:1235-1245 (2000).

Kim S: Congenital hypogonadotropic hypogonadism and Kallmann syndrome: past, present, and future. Endocrinol Metab 30:456 (2015).

- LeCouter J, Lin R, Tejada M, Frantz G, Peale F, et al: The endocrine-gland-derived VEGF homologue Bv8 promotes angiogenesis in the testis: localization of Bv8 receptors to endothelial cells. Proc Natl Acad Sci USA 100: 2685-2690 (2003).

Lund SP, Nettleton D, McCarthy DJ, Smyth GK: Detecting differential expression in RNA-sequence data using quasi-likelihood with shrunken dispersion estimates. Stat Appl Genet Mol Biol 11 (2012).

-Martin C, Balasubramanian R, Dwyer AA, Au MG, Sidis Y, et al: The role of the prokineticin 2 pathway in human reproduction: evidence from the study of human and murine gene mutations. Endocr Rev 32:1-22 (2010).
McGillivray SM, Bailey JS, Ramezani R, Kirkwood BJ, Mellon PL: Mouse GnRH receptor gene expression is mediated by the LHX3 homeodomain protein. Endocrinology 146: 2180-2185 (2005).

Merchenthaler I, Görcs T, Sétáló G, Petrusz P, Flerkó B: Gonadotropin-releasing hormone $(\mathrm{GnRH})$ neurons and pathways in the rat brain. Cell Tissue Res 237:15-29 (1984).

Miller DC, Saigal CS, Litwin MS: The demographic burden of urologic diseases in America. Urol Clin North Am 36:11-27 (2009).

Robinson MD, McCarthy DJ, Smyth GK: edgeR: a bioconductor package for differential expression analysis of digital gene expression data. Bioinformatics 26:139-140 (2010).

-Sadie H, Styger G, Hapgood J: Expression of the mouse gonadotropin-releasing hormone receptor gene in aT3-1 gonadotrope cells is stimulated by cyclic $3^{\prime}, 5^{\prime}$-adenosine monophosphate and protein kinase $\mathrm{A}$, and is modulated by steroidogenic factor-1 and Nur77. Endocrinology 144:1958-1971 (2003).

-Samson M, Peale FV, Frantz G, Rioux-Leclercq N, Rajpert-De Meyts E, Ferrara N: Human endocrine gland-derived vascular endothelial growth factor: expression early in development and in Leydig cell tumors suggests roles in nor$\mathrm{mal}$ and pathological testis angiogenesis. J Clin Endocrinol Metab 89:4078-4088 (2004).

-Schneider-Maunoury S, Topilko P, Seitanidou T, Levi G, Cohen-Tannoudji M, et al: Disruption of Krox-20 results in alteration of rhombomeres 3 and 5 in the developing hindbrain. Cell 75:1199-1214 (1993).

Seguchi H, Hadziselimovic F: Ultramicroscopic studies on the seminiferous tubule in children from birth to puberty. I. Spermatogonia development. Verh Anat Ges 68:133-148 (1974)

- Sinisi AA, Asci R, Bellastella G, Maione L, Esposito $\mathrm{D}$, et al: Homozygous mutation in the prokineticin-receptor2 gene (Val274Asp) presenting as reversible Kallmann syndrome and persistent oligozoospermia: case report. Hum Reprod 23:2380-2384 (2008).

-Swiatek PJ, Gridley T: Perinatal lethality and defects in hindbrain development in mice homozygous for a targeted mutation of the zinc finger gene Krox20. Genes Dev 7:2071-2084 (1993).

Topilko P, Schneider-Maunoury S, Levi G, Baron-Van Evercooren A, Chennoufi AB, et al: Krox-20 controls myelination in the peripheral nervous system. Nature 371:796-799 (1994).

- Tourtellotte WG, Nagarajan R, Auyeung A, Mueller C, Milbrandt J: Infertility associated with incomplete spermatogenic arrest and oligozoospermia in Egr4-deficient mice. Development 126:5061-5071 (1999).

Tourtellotte WG, Nagarajan R, Bartke A, Milbrandt J: Functional compensation by Egr4 in Egr1-dependent luteinizing hormone regulation and Leydig cell steroidogenesis. Mol Cell Biol 20:5261-5268 (2000).
Gene Expression in Cryptorchidism with Defective Mini-Puberty 
Tremblay JJ, Drouin J: Egr-1 is a downstream effector of GnRH and synergizes by direct interaction with Ptx1 and SF-1 to enhance luteinizing hormone beta gene transcription. Mol Cell Biol 19:2567-2576 (1999).

Valdes-Socin H, Almanza MR, Fernández-Ladreda MT, Debray FG, Bours V, Beckers A: Reproduction, smell, and neurodevelopmental disorders: genetic defects in different hypogonadotropic hypogonadal syndromes. Front Endocrinol (Lausanne) 5:1-8 (2014).

Verkauskas G, Malcius D, Eidukaite A, Vilimas C, Dasevicius D, et al: Prospective study of histological and endocrine parameters of gonadal function in boys with cryptorchidism. J Pediatr Urol (2016) Epub ahead of print. von Elm E, Altman DG, Egger M, Pocock SJ, Gøtzsche PC, Vandenbroucke JP: The strengthening the reporting of observational studies in epidemiology (STROBE) statement: guidelines for reporting observational studies. Int J Surg 12:1495-1499 (2014).

Wechselberger C, Puglisi R, Engel E, Lepperdinger G, Boitani C, Kreil G: The mammalian homologues of frog Bv8 are mainly expressed in spermatocytes. FEBS Lett 462:177-181 (1999).

Wei X, Sasaki M, Huang H, Dawson VL, Dawson TM: The orphan nuclear receptor, steroidogenic factor 1 , regulates neuronal nitric oxide synthase gene expression in pituitary gonadotropes. Mol Endocrinol 16:2828-2839 (2002).
Winter JS, Hughes IA, Reyes FI, Faiman C: Pituitary gonadal relations in infancy: II. Patterns of serum gonadal steroid concentrations in man from birth to two years of age. J Clin Endocrinol Metab 42:679-686 (1976).

-Wray S, Hoffman G: A developmental study of the quantitative distribution of LHRH neurons within the central nervous system of postnatal male and female rats. J Comp Neurol 252:522-531 (1986)

Xie H, Cherrington BD, Meadows JD, Witham EA, Mellon PL: Msx1 homeodomain protein represses the $\alpha \mathrm{GSU}$ and $\mathrm{GnRH}$ receptor genes during gonadotrope development. Mol Endocrinol 27:422-436 (2013). 